\section{SAÚDEE AMBIENTE}

v.7 $\cdot$ N.3 • Junho -2019

ISSN Digital: 2316-3798

ISSN Impresso: 2316-3313

DOI: 10.17564/2316-3798.2019v7n3p133-142 ORCID: 2-7779-5126

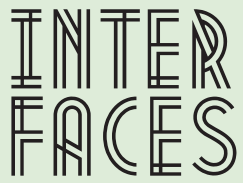

CIENTÍFICAS

\title{
LEISHMANIOSE VISCERAL: PERFIL EPIDEMIOLÓGICO DOS CASOS NO MUNICÍPIO DE MACEIÓ, ALAGOAS NO PERÍODO DE 2011 A 2016
}

LEISHMANIOSE VISCERAL: EPIDEMIOLOGICAL PROFILE OF THE CASES IN THE MUNICIPALITY OF MACEIÓ, ALAGOAS NO PERIOD FROM 2011 TO 2016

\section{LEISHMANIOSIS VISCERAL: PERFIL EPIDEMIOLÓGICO DE LOS CASOS EN EL MUNICIPIO DE MACEIÓ, ALAGOAS EN EL PERÍODO DE 2011 A 2016}

Anacássia Fonseca de Lima $^{1}$ Allana Fernanda Sena dos Santos ${ }^{2}$ Thaís Rafaela Santos Pinto Calheiros ${ }^{3}$ Antônio Fernando Silva Xavier Junior ${ }^{4}$ Sabrina Gomes de Oliveira ${ }^{5}$

\section{RESUMO}

A Leishmaniose Visceral é uma doença zoonótica, causada por uma espécie de protozoário do gênero Leishmania, transmitida por insetos denominados flebotomíneos. Também conhecida como calazar, que além de afetar a saúde humana também pode comprometer a saúde de outros animais como o cão doméstico. Trata-se de estudo epidemiológico transversal de abordagem quantitativa, que avaliou os casos de leishmaniose visceral registrados em Maceió, Alagoas no período de 2011 a 2016. A coleta dos dados ocorreu no Sistema de Informações de Agravos de Notificação. Foi utilizado o coeficiente de incidência por 100 mil habitantes no mesmo período e razão de risco da ocorrência dos casos entre as categorias de cada grupo. A incidência de casos de leishmaniose visceral, para o período, foi de 3,0 casos para cada 100.000 habitantes. Na análise do gênero o sexo masculino apresentou maior incidência com 4,3 casos para cada 100.000 habitantes, nas faixas etárias de 0 a 4 e 5 a 9 anos a taxa de incidência foi de 12,6 e 5,1 para cada 100.000 habitantes respectivamente. A situação observada no município de Maceió poderá servir de referência para novas pesquisas, avaliações de indicadores epidemiológicos, além de contribuir com as estratégias de controle da doença na população.

\section{PALAVRAS-CHAVE}

Epidemiologia. Leishmaniose Visceral. Incidência. 


\section{ABSTRACT}

Visceral Leishmaniasis is a zoonotic disease, caused by a protozoan species of the genus Leishmania, transmitted by insects called sandflies. Also known as calazar, which in addition to affecting human health can also compromise the health of other animals like the domestic dog. This is a cross-sectional epidemiological study with a quantitative approach, which evaluated the cases of visceral leishmaniasis recorded in Maceió, Alagoas from 2011 to 2016. The data collection took place in the Information System of Notifiable Diseases. The incidence coefficient per 100,000 inhabitants was used in the same period and the risk ratio of the occurrence of cases among the categories of each group. The incidence of cases of visceral leishmaniasis for the period was 3.0 cases per 100,000 inhabitants. In the gender analysis, males presented a higher incidence with 4.3 cases per 100,000 inhabitants, in the age groups 0 to 4 and 5 to 9 years the incidence rate was 12.6 and 5.1 for each 100,000 inhabitants respectively. The situation observed in the municipality of Maceió can serve as a reference for new research, evaluations of epidemiological indicators, and contribute to the strategies of disease control in the population.

\section{KEYWORDS}

Epidemiology. Visceral Leishmaniasis. Incidence.

\section{RESUMEN}

La Leishmaniosis Visceral es una enfermedad zoonótica, causado por una especie de protozoario del género Leishmania, transmitida por insectos denominados flebotomíneos. También conocida como kala-azar, que además de afectar la salud humana también puede comprometer la salud de otros animales como el perro doméstico. Se trata de un estudio epidemiológico transversal de abordaje cuantitativo, que evaluó los casos de leishmaniosis viscerales registrados en Maceió, Alagoas en el período de 2011 a 2016. La recolección de los datos ocurrió en el Sistema de Información de Agravios de Notificación. Se utilizó el coeficiente de incidencia por 100 mil habitantes en el mismo período y razón de riesgo de la ocurrencia de los casos entre las categorías de cada grupo. La incidencia de casos de leishmaniosis visceral, para el período, fue de 3,0 casos por cada 100.000 habitantes. En el análisis del género el sexo masculino presentó mayor incidencia con 4,3 casos por cada 100.000 habitantes, en las franjas de edad de 0 a 4 y 5 a 9 años la tasa de incidencia fue de 12,6 y 5,1 por cada 100.000 habitantes respectivamente. La situación observada en el municipio de Maceió podría servir de referencia para nuevas investigaciones, evaluaciones de indicadores epidemiológicos, además de contribuir con las estrategias de control de la enfermedad en la población. 


\section{PALABRAS CLAVE}

Epidemiología, Leishmaniosis Visceral, Incidencia.

\section{INTRODUCÇÃO}

A Leishmaniose Visceral (LV) é uma doença zoonótica, causada por uma espécie de protozoário do gênero Leishmania, transmitida por insetos (vetores) denominados flebotomíneos, conhecidos popularmente como mosquito palha, tatuquira, birigui (ROCHA et al., 2015; BARBOSA; COSTA, 2013). No Brasil, a principal espécie responsável pela transmissão da LV é Lutzomyia longipalpis e o agente etiológico é Leishmania infantum chagasi (LUCENA; MEDEIROS, 2018; BRASIL, 2010).

Conhecida como calazar, a LV é uma doença que afeta outros animais como raposas (Lycalopex vetulus e Cerdocyon thous) e marsupiais (Didelphis albiventris) apontados como reservatórios silvestres (BRASIL, 2017). O cão doméstico (Canis familiaris) é considerado o principal reservatório do parasito, podendo ser um perigo potencial para a transmissão para os seres humanos (LUCENA; MEDEIROS, 2018).

Possui caráter endêmico em cinco continentes, com casos relatados em 54 países de regiões tropicais e subtropicais. Mais de $90 \%$ dos casos ocorridos no mundo localiza-se em países como Bangladesh, Índia, Sudão, Sudão do Sul, Etiópia e Brasil (MARCONDES; ROSSI, 2013). É considerada a forma mais grave da doença, podendo acarretar óbitos se não tratada adequadamente (CARDIM et al., 2016).

No Brasil a doença afeta cinco regiões em 20 estados. Observa-se maior incidência no nordeste com aproximadamente $45 \%$ de todos os casos, seguida pelas regiões Sudeste, Norte e Centro-Oeste (PONTE et al., 2011). O número aproximado de casos ocorridos no Brasil anualmente é de 3.500. A taxa de incidência da LV atinge 20,4 casos por 100.000 habitantes em algumas regiões dos estados do Nordeste, como Paraíba, Piauí, Maranhão e Bahia (BRASIL, 2014; LUCENA; MEDEIROS, 2018).

Considerada uma protozoonose (doença causada por protozoário) cujo espectro clínico há variações desde manifestações clínicas discretas até graves. A evolução prolongada da doença é caracterizada por febre irregular de longa duração, distúrbios gastrointestinais, emagrecimento, sonolência, prostração, palidez cutaneomucosa, que confere um aspecto escurecido à pele dos indivíduos caucasianos. Associa-se à exuberante hepatoesplenomegalia, anemia, leucopenia e trombocitopenia. Podem ocorrer manifestações hemorrágicas como epitaxes e sangramento gengival e os sintomas respiratórios lembram um resfriado comum (AGUIAR; RODRIGUES, 2017).

Muitos infectados apresentam a forma branda da doença ou são assintomáticos, no entanto se não houver um tratamento adequado à infecção poderá levar o indivíduo a óbito (BRASIL, 2010).

Este estudo tem por objetivo caracterizar o perfil epidemiológico dos casos de LV no município de Maceió no período de 2011 a 2016; acredita-se que ele contribua nos diálogos da equipe de saúde e gestores envolvidos no planejamento das ações em saúde. 


\section{MÉTODO}

Trata-se de estudo epidemiológico transversal de abordagem quantitativa, no âmbito dos registros de casos de LV em Maceió, Alagoas no período de 2011 a 2016 coletados por meio do Sistema de Informações de Agravos de Notificação (SINAN) na plataforma de informações de saúde - TABNET do Ministério da Saúde, em que são disponibilizados os números de casos de doenças de uma população por região ou estado.

De acordo com a Portaria $n^{\circ}$ 4, de 28 de Setembro de 2017, o SINAN é alimentado, pela notificação e investigação de casos de doenças e agravos que constam da lista nacional de doenças de notificação compulsória; ele orienta as ações de controle de doenças, fornece registros, fluxo de informações e tabulação de dados, sobre casos de notificação compulsória, é considerado um modelo de integração sistêmica com grande potencial de inserção e difusão de novas tecnologias na rede de serviços de saúde (ALVES; FONSECA, 2018).

A Lista de Tabulação no SINAN é apresentada na plataforma de informações de saúde - TABNET, de acordo com volume I da Classificação Internacional de Doenças (CID-10) na qual é a nomenclatura Leishmaniose Visceral segundo CID -10 (capítulo 1- algumas doenças infecciosas e parasitárias com o código A00-B99).

As variáveis estudadas foram: sexo (masculino e feminino), faixa etária (0-4; 5-9; 10-14; 15-19; 20-29; 30-39; 40-49; 50-59; 60-69; 70-79 e $\geq 80$ anos de idade), critério de confirmação (laboratorial e clínico epidemiológico) e evolução do caso (cura, abandono, óbito por LV, óbito por outra causa). As estimativas da população para os cálculos de incidência foram obtidas do sítio do Instituto Brasileiro de Geografia e Estatística (IBGE), na Seção “Projeção da População” (BRASIL, 2018).

Os dados foram organizados e armazenados em planilha eletrônica do Microsoft Excel 2007. Na análise descritiva, o número de casos foi categorizado de acordo com as variáveis coletadas na base. Para fins de entendimento foi utilizado o Coeficiente de incidência (CP) por 100 mil habitantes no mesmo período no qual é utilizado para obter o número total de casos existentes numa determinada população num determinado período. Após a obtenção dos valores, foi calculada a média do coeficiente, a fim de representar o período estudado. A partir de então, foi procedido o cálculo da razão de risco R1/R2 da ocorrência dos casos entre as categorias de cada grupo. Os dados correspondentes ao critério de confirmação e evolução do caso foi utilizado o cálculo proporcional para caracterizar o período.

A pesquisa atendeu aos preceitos éticos e legais conforme determinação da Resolução 466/12 do Conselho Nacional de Saúde. Nesse sentido, considerando que o estudo foi realizado com dados provenientes do Sistema de Informações de Agravos de Notificação (SINAN) na plataforma de informações de saúde - TABNET do Ministério da Saúde (http://www2.datasus.gov.br), dados secundários de domínio público, sem riscos à população e sem identificação nominal dos indivíduos, tornou-se desnecessária a autorização do comitê de ética e pesquisa. 


\section{RESULTADOS}

No período estudado, foram registrados 182 casos de LV no município. A incidência de casos de LV, para o período, foi de 3,0 para cada 100.000 habitantes. No ano de 2015 foram registrados 41 casos, com incidência de 4,0 para cada 100.000 habitantes, considerado um maior índice em relação aos anos anteriores (TABELA 1).

Tabela 1 - Distribuição de frequência dos casos e coeficiente de incidência média por LV, no período de 2011 a 2016 em Maceió

\begin{tabular}{ccc}
\hline Ano & No de casos $^{\text {col }}$ & C. \\
\hline 2011 & 31 & 3,2 \\
2012 & 31 & 3,1 \\
2013 & 23 & 2,3 \\
2014 & 35 & 3,5 \\
2015 & 41 & 4,0 \\
2016 & 21 & 2,1 \\
TOTAL & 182 & \\
Coeficiente Médio & & 3,0 \\
\hline
\end{tabular}

* C.I: Coeficiente de Incidência expresso para cada 100.000 habitantes.

Fonte: SIH/SUS-DATASUS. Ministério da Saúde. Disponível em 18/09/2018. Dados sujeitos a revisão.

Quanto ao sexo, percebe-se que o registro mais frequente foi referente ao sexo masculino, com incidência de 4,3 para cada 100.000 habitantes. De acordo com a análise o risco para homens adoecerem é de 2,3 quando comparado ao risco no sexo feminino (TABELA 2).

Tabela 2 - Coeficiente de incidência média e razão de risco por LV segundo sexo no período de 2011 a 2016 em Maceió

\begin{tabular}{cccc}
\hline Sexo & No de casos & $*$ C.I & Razão de Risco \\
\hline Masculino & 122 & 4,3 & 2,3 \\
Feminino & 60 & 1,9 & 1,0 \\
Total & 182 & & \\
Coeficiente Médio & & 3,1 & \\
\hline
\end{tabular}

* C.P: Coeficiente de incidência expresso para cada 100.000 habitantes.

Fonte: SIH/SUS-DATASUS. Ministério da Saúde. Disponível em 18/09/2018. Dados sujeitos a revisão. 
$\mathrm{Na}$ análise da faixa etária, as idades de 0 a 4 anos e 5 a 9 anos, tida como a primeira infância a incidência foi de 12,6 e 5,1, para cada 100.000 habitantes. As mesmas apresentaram uma razão de risco de 8,6 e 3,5 respectivamente, quando comparadas à faixa etária de 40 a 59 anos. Ressaltando que durante o período a faixa etária a partir de 70 anos, não obteve registros de casos por LV (TABELA 3).

Tabela 3 - Distribuição do número de casos, coeficiente de incidência média por LV e razão de risco, segundo faixa etária no período de 2011 a 2016 em Maceió

\begin{tabular}{cccc}
\hline Faixa etária & No de internações & C.I* & Razão de risco \\
\hline 0 a 4 anos & 61 & 12,6 & 8,6 \\
5 a 9 anos & 26 & 5,1 & 3,5 \\
10 a 14 anos & 20 & 3,8 & 2,6 \\
15 a 19 anos & 16 & 4,7 & 3,2 \\
20 a 39 anos & 35 & 1,7 & 1,1 \\
40 a 59 anos & 20 & 1,5 & 1,0 \\
60 a 69 anos & 3 & 2,0 & 1,3 \\
TOTAL & 182 & & \\
Coeficiente médio & & 4,5 &
\end{tabular}

${ }^{*}$ C.I: Coeficiente de incidência expresso para cada 100.000 habitantes.

Fonte: SIH/SUS-DATASUS. Ministério da Saúde. Disponível em 18/09/2018. Dados sujeitos a revisão.

Quanto ao critério de confirmação, o laboratorial atingiu 74,7\% dos casos confirmados. 0 caracterizado como clínico-epidemiológico, obteve $25,3 \%$ dos registros durante o período (TABELA 4).

Tabela 4 - Distribuição proporcional de casos de LV, segundo critério de confirmação da patologia no período de 2011 a 2016 em Maceió

\begin{tabular}{ccc}
\hline Critério de confirmação & Casos & *\% \\
\hline Laboratorial & 136 & 74,7 \\
Clínico-epidemiológico & 46 & 25,3 \\
Total & 182 & 100 \\
\hline
\end{tabular}

* \%: Casos de LV segundo critério de confirmação expresso em porcentagem

Fonte: SIH/SUS-DATASUS. Ministério da Saúde. Acesso em 18/09/2018. Dados sujeitos a revisão.

De acordo com a evolução do caso, ignorado/branco registrou $51,6 \%$, seguido por cura $31,3 \%$, óbito por LV 7,7\%, óbito por outra causa 5,5\%, transferência 2,7\%, abandono 1,1\%. Entretanto, 0 
número de casos com registro caracterizado como ignorado prevaleceu perante os demais, o que dificulta a análise (TABELA 5).

Tabela 5 - Distribuição proporcional de casos de LV, segundo evolução do caso no período de 2011 a 2016 em Maceió

\begin{tabular}{ccc}
\hline Evolução do caso & $\mathbf{N}^{\mathbf{0}}$ de casos & $* \%$ \\
\hline Ign/Branco & 94 & 51,6 \\
Cura & 57 & 31,3 \\
Abandono & 2 & 1,1 \\
Óbito por LV & 14 & 7,7 \\
Óbito por outra causa & 10 & 5,5 \\
Transferência & 5 & 2,7 \\
Total & 182 & 100 \\
\hline
\end{tabular}

* \%: casos de LV segundo evolução do caso expresso em porcentagem.

Fonte: SIH/SUS-DATASUS. Ministério da Saúde. Acesso em 18/09/2018. Dados sujeitos a revisão.

\section{DISCUSSÃO}

Na análise dos resultados em Maceió obteve 182 casos de LV no período. De acordo com Lucena e Medeiros (2018) o número de casos de LV humana em Alagoas se contrasta em relação ao restante dos estados que apresentaram uma incidência de casos elevada no período do estudo, refletindo diretamente no município.

Quanto ao gênero, revelou predomínio no sexo masculino. Segundo dados da pesquisa de Alves e Fonseca (2018) 69,5\% dos casos de LV foi em homens. Apesar da importância epidemiológica e de não estar totalmente esclarecido, o risco de adoecimento em homens pode ser justificado principalmente pelo fato da exposição ser maior em suas atividades diárias em locais suscetíveis à transmissão (GOES et al., 2012; ALVES; FONSECA, 2018).

A faixa etária com maior ocorrência de casos notificados está na primeira infância. Estes resultados corroboram os dados de um estudo realizado, em 2012 no Estado do Maranhão, que obteve $32,75 \%$ de casos em crianças menores de 4 anos e 13,54\% em crianças menores de 10 anos (SALES et al., 2017). Tal achado justifica-se pelo contato frequente das crianças com animais, além de seu estado imunológico ainda em formação (SOUSA, 2018).

Um estudo sobre letalidade realizado no estado de Alagoas revelou que o índice de letalidade de LV aumentou nos anos de 2009 a 2012, esses resultados ocorreram principalmente em pessoas do sexo masculino, em crianças de cinco a nove anos, essa faixa etária apresentou um número maior de casos, porém, não houve nenhum óbito (ROCHA et al., 2015). 
A LV acomete pessoas de todas as faixas etárias, porém a maioria dos casos registrados ocorre em crianças com menos de 10 anos. Não há diferenças na suscetibilidade entre pessoas de raças ou idades distintas, entretanto, o índice da doença é maior em crianças menores, principalmente entre um e quatro anos de idade. Em algumas cidades estudadas existe uma tendência de modificação na distribuição dos casos por grupo etário, com ocorrência de altas taxas também no grupo de adultos jovens (COSTA, 2018).

Várias doenças que complicam com a hepatoesplenomegalia febril podem ser confundidas com a LV. Destaca-se entre as doenças não transmissíveis: neoplasias, como o linfoma. Entre as transmissíveis: febre tifoide, esquistossomose, toxoplasmose disseminada, histoplasmose entre outras. Tornando o diagnóstico clínico-epidemiológico insuficiente para o início do tratamento. Entretanto tal diagnóstico é realizado para os pacientes com suspeita clínica, residente em área de transmissão, onde a confirmação laboratorial pode não ser realizada (COSTA, 2018).

Quanto à evolução da doença, nos estudos realizados, observa-se uma porcentagem elevadade cura em pacientes acometidos por LV e baixa para o óbito por essa patologia (BARBOSA, 2013; SANTOS et al., 2017). No entanto outros estudos relatam que há uma reduçãono índice de cura, porém a letalidade se torna alta por acometer as crianças (CAVALCANTE; VALE, 2014). Isso sugere que um número significativo de pacientes seguem o tratamento e, consequentemente o número de óbitos é abaixo do que se espera para a doença.

\section{CONCLUSÃO}

De acordo com os dados coletados as variáveis de LV predominantes foram: 2015 com o maior número de casos no período, o sexo masculino, crianças de 0 a 4 anos e 5 a 9 anos, critério de confirmação laboratorial, evolução o caso com a prevalência maior no ignorado/branco.

Apesar de a grande maioria dos casos evoluírem para a cura, a incidência em homens e crianças é elevada, tendo sido observado um aumento preocupante da doença no ano de 2015. Tal situação no município poderá servir de referência para novas pesquisas sobre o tema, que permitam comparar as áreas de risco e avaliar os mais variados indicadores epidemiológicos, além de contribuir nas atuais estratégias de controle da doença na população com medidas de educação em saúde entre outras.

\section{REFERÊNCIAS}

AGUIAR, P. F.; RODRIGUES, R. K. Leishmaniose visceral no Brasil: artigo de revisão. Rev. Unimontes Cient, v. 19, n.1, p. 191-204, 2017.

ALVES, W. A.; FONSECA, D. S. Leishmaniose visceral humana: estudo do perfil clínicoepidemiológico na região leste de minas gerais, Brasil. J Health Biol Sci, v. 6, n. 2, p. 133-139, 2018. 
BARBOSA, I. R. Epidemiologia da Leishmaniose Visceral no estado do Rio Grande do Norte, Brasil. Rev Epidemiol Control Infect, v. 3, n. 1, p. 17-21, 2013.

BARBOSA, I. R.; COSTA, I. C. C. Aspectos clínicos e epidemiológicos da leishmaniose visceral em menores de 15 anos no estado do Rio Grande do Norte, Brasil. Scientia Med, v. 23, n. 1, p. 5-11, 2013.

BRASIL. Ministério da Saúde. Projeção da população das unidades da federação por sexo e grupos de idade: 2000-2030 - Nota Técnica, 2018.

BRASIL. Ministério da Saúde. Secretaria de Vigilância em Saúde. Departamento de Vigilância Epidemiológica. Doenças infecciosas e parasitárias: guia de bolso. 8. ed. Brasília: Ministério da Saúde, 2010.

BRASIL. Ministério da Saúde. Secretaria de Vigilância em Saúde. Departamento de Vigilância Epidemiológica. Manual de vigilância e controle da leishmaniose visceral. Brasília: Ministério da Saúde, 2014.

BRASIL. Ministério da Saúde. Portaria de consolidação nº 4, de 28 de setembro de 2017. Brasília: Ministério da Saúde, 2017.

CARDIM, F. M. et al. Leishmaniose visceral no estado de São Paulo, Brasil: análise espacial e espaçotemporal. Rev Saúde Pública, v. 50, a. 48, 2016.

CAVALCANTE, I. J. M.; VALE, M. R. Aspectos epidemiológicos da leishmaniose visceral (calazar) no Ceará no período de 2007 a 2011. Rev Bras Epidemiol, v. 17, n. 4, p. 911-924, 2014.

COSTA, J. N. G. Avaliação do sistema de vigilância da leishmaniose visceral humana no Brasil, 2011 - 2015. 2018. 65f. Dissertação (Mestrado em Ciências da Saúde) - Universidade Federal do Tocantins, Palmas, 2018.

GOES, M. A. O. G. et al. Série temporal de leishmaniose visceral em Aracaju, Sergipe, Brasil (1999 a 2008): aspectos humanos e caninos. Rev Bras Epidemiol, v. 15, n. 2, p. 298-307, 2012.

LUCENA, R. V.; MEDEIROS, J. S. Caracterização epidemiológica da leishmaniose visceral humana no nordeste brasileiro entre 2010 e 2017. J Biol Pharm Agr Manag, v. 14, n. 4, p. 285-298, 2018.

MARCONDES, M.; ROSSI, C. N. Leishmaniose Visceral no Brasil. Braz J Vet Res Anim Sci, v. 50, n. 5, p. 341-352, 2013.

PONTE, C. B. et al. Fatores associados à infecção por Leishmania chagasi em uma área endêmica em raposa, Estado do Maranhão, Brasil. Rev Soc Bras Med Trop, v. 44, n. 6, p. 712-721, 2011. 
ROCHA, T. J. M. et al. Perfil epidemiológico relacionado aos casos de letalidade por leishmaniose visceral em Alagoas: uma análise entre os anos de 2007 a 2012. Rev Ciênc Farm Básica Apl, v. 36, n. 1, p. 17-20, 2015.

SALES, D. P. et al. Aspectos epidemiológicos da Leishmaniose Visceral Canina e Humana no estado do Maranhão, Brasil (2009-2012). Rev Bras Cien Vet, v. 24, n. 3, p. 144-150, 2017.

SANTOS, G. M. Aspectos epidemiológicos e clínicos da leishmaniose visceral no estado do Piauí, Brasil. C\&D-Rev Eletr FAINOR, v. 10, n. 2, p. 142-153, 2017.

SOUSA, N. A. et al. Perfil epidemiológico dos casos de leishmaniose visceral em Sobral-CE de 2011 a 2015, SANARE, v. 17, n. 1, p. 51-57, 2018.

1 Mestre em Patologia - UFPE; Professora Adjunta do Centro Universitário Tiradentes - UNIT/AL; Professora Titular do Centro Universitário CESMAC/AL.

E-mail: cassialima3@hotmail.com

2 Acadêmica do Curso de Enfermagem, Centro Universitário Tiradentes - UNIT/AL. E-mail: allanafss@outlook.com

3 Acadêmica do Curso de Enfermagem, Centro Universitário Tiradentes - UNIT/AL. E-mail: thais.pinto92@hotmail.com

4 Mestre em Nutrição - UFAL; Professor Adjunto do Centro Universitário Tiradentes - UNIT/AL.

E-mail: antoniofernando_jr@yahoo.com.br

5 Doutora em Ciências, Faculdade de Medicina - USP; Professora Titular do Centro Universitário Tiradentes - UNIT/ AL. E-mail: sabrinaoliveiramedvet@yahoo.com.br

\section{Como citar este artigo:}

$X X X X X X X X X X X X X X X X X X X X X X X X X X X X X X X X X X$

$X X X X X X X X X X X X X X X X X X X X X X X X X X X X X X X X X X$ $x X X X X X X X X X X X X X X X X X X X X X X X X X X X X X X X X$

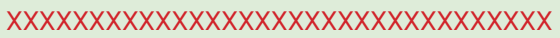
$x X X X X X X X X X X X X X X X X X X X X X X X X X X X X X X X X X$

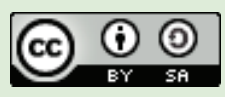

Este artigo é licenciado na modalidade acesso abertosob a Atribuição-Compartilhalgual CC BY-SA

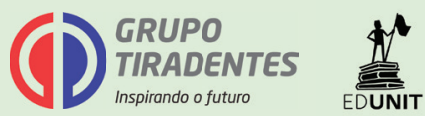

